\title{
6 Kurzfassungen der Kostensensiblen Leitlinien
}

\author{
BMBF-Forschungsverbund „Allokation“
}

Das vorliegende Kapitel präsentiert die im interdisziplinären, vom Bundesministerium für Bildung und Forschung (BMBF) finanzierten Forschungsverbund „Allokation“ entwickelten Kurzfassungen der Kostensensiblen Leitlinien (KSLL), wie sie dann auch als Grundlage für die empirischen Studien benutzt wurden (s. Kap. 9 u. 10). Vorangestellt ist die allgemeine Einführung in die KSLL, die den Gesprächsteilnehmern zusammen mit den exemplarischen KSLL zur Verfügung gestellt wurde. Für die Interviews mit den Patienten wurde eine für medizinische Laien besser verständliche Version der KSLL für Medikamente-freisetzende Stents (drug eluting stents, DES) erstellt. Auch die allgemeine Einführung zu den KSLL wurde entsprechend angepasst.

\subsection{Kostensensible Leitlinien (KSLL) als Instrument einer expliziten Allokation knapper Gesundheitsressourcen: zur Einführung}

\subsubsection{Hintergrund}

Nach weithin geteilter Auffassung werden der medizinisch-technische Fortschritt und der demographische Wandel die Nachfrage nach Gesundheitsleistungen weiter in die Höhe treiben. Dem stehen in der Gesetzlichen Krankenversicherung (GKV) begrenzt verfügbare finanzielle Ressourcen gegenüber. 
Aufgrund der zunehmenden Diskrepanz zwischen medizinisch Machbarem und solidarisch Finanzierbarem werden Leistungseinschränkungen unausweichlich. Im interdisziplinären, vom Bundesministerium für Bildung und Forschung (BMBF) finanzierten Forschungsverbund „Allokation“ haben Wissenschaftler aus den Bereichen der Medizinethik, der Gesundheitsökonomie und des Medizinrechts untersucht, wie diese Leistungseinschränkungen in einer medizinisch rationalen, ökonomisch sinnvollen und ethisch wie rechtlich vertretbaren Art und Weise durchgeführt werden können.

\subsubsection{Explizite vs. implizite Leistungsbegrenzungen}

Leistungsbegrenzungen können auf zwei verschiedenen Ebenen durchgeführt werden. Explizite Leistungsbegrenzungen erfolgen „oberhalb“ der individuellen Arzt-Patienten-Beziehung nach ausdrücklich festgelegten, allgemein verbindlichen Kriterien. Bei impliziten Leistungsbegrenzungen erfolgt die Zuteilung hingegen nicht nach allgemein vorgegebenen Regeln, sondern jeweils im Einzelfall durch Ärzte. Sie resultieren aus begrenzten Budgets oder finanziellen Anreizen (z.B. Fallpauschalen-Vergütung im Krankenhaus).

\subsubsection{Ethische Rechtfertigung der Kostensensiblen Leitlinien}

Aus ethischer Sicht weisen explizite Leistungsbegrenzungen gegenüber impliziten verschiedene Vorteile auf: Sie sind transparent, konsistent, medizinisch rationaler und durch die Gleichbehandlung der Patienten gerechter, überdies entlasten sie die Arzt-Patient-Beziehung und erlauben eine simultane Steuerung von Qualität und Kosten der Versorgung. Mit den Kostensensiblen Leitlinien (KSLL) hat der Forschungsverbund „Allokation“ ein Modell entwickelt, wie explizite Leistungsbegrenzungen in der Praxis umgesetzt werden können. Den KSLL liegt dabei folgende ethische Überlegung zugrunde: Wenn Leistungsbegrenzungen unausweichlich sind - und davon müssen wir zumindest im Bereich der GKV ausgehen -, sollten diese so durchgeführt werden, dass den Patienten ein möglichst geringer (Zusatz-)Nutzen vorenthalten wird. Man sollte folglich auf diejenigen Maßnahmen verzichten, die dem Patienten bei relativ hohen Kosten einen nur geringen Zusatznutzen bieten. Die freiwerdenden Ressourcen können dann anderen Patienten mit einem größeren Nutzengewinn (oder einer schwerwiegenderen Erkrankung) zugutekommen. Bei der Erstellung von KSLL wird nun auf der Crundlage der wissenschaftlichen Evidenz zur Effektivität und Kosteneffektivität herausgearbeitet, welche Patientengruppen mehr oder weniger von einer bestimmten medizinischen Maßnahme profitieren. Die KSLL schränkt dann die Indikation, d.h. den Einsatzbereich der Maßnahme auf diejenigen Patienten ein, die den größten Nutzengewinn von ihr haben. Patienten mit einem nur geringen Nutzengewinn müssen - sofern verfügbar - auf alternative Verfahren ausweichen. Für die 
6.1 Kostensensible Leitlinien (KSLL) als Instrument einer expliziten Allokation knapper Gesundheitsressourcen: zur Einführung

ethische Legitimation ist neben der inhaltlichen Begründung entscheidend, dass die KSLL in einem transparenten und fairen Entscheidungsverfahren erstellt wurde.

\subsubsection{Methodik der Kostensensiblen Leitlinien}

Bei der Erstellung von KSLL sind zunächst Patienten-Subgruppen zu identifizieren, die einen unterschiedlich großen Nutzen von den jeweiligen medizinischen Maßnahmen haben. In einem zweiten Schritt wird dann die Kosteneffektivität für die verschiedenen Subgruppen bestimmt. Bei gleich bleibenden Behandlungskosten ist die Kosteneffektivität schlechter, je geringer der Nutzengewinn für die Patienten gegenüber der nächstgünstigeren Alternative ist. Die KSLL legt nun explizit fest, welche Patienten-Subgruppen die Maßnahmen erhalten sollen: Übersteigt das Verhältnis von Kosten und Nutzen einen bestimmten Grenzwert, wird die Maßnahme für die betreffende Patienten-Subgruppe nicht mehr von der GKV finanziert. Patienten mit einem großen Nutzengewinn erhalten die Maßnahme auf GKV-Kosten, während Patienten, die von der Maßnahme nur wenig profitieren würden, auf eine kostengünstigere Behandlungsmöglichkeit ausweichen oder die Maßnahme privat finanzieren müssen.

KSLL bieten die Möglichkeit, Nutzen und Kosten medizinischer Maßnahmen explizit gegeneinander abzuwägen und schaffen damit die Voraussetzung für einen medizinisch rationalen (im Sinne von evidenzbasierten), effizienten und ethisch vertretbaren Einsatz der knapper werdenden Mittel. Im derzeitigen System der GKV müssten KSLL vom Gemeinsamen Bundesausschuss (GBA) als grundsätzlich verbindliche Vorgaben erlassen werden, von denen aber in begründeten Ausnahmefällen auch abgewichen werden kann.

Um Stärken und Schwächen der Instrumente besser einschätzen zu können, wurden im Forschungsverbund zwei exemplarische KSLL für implantierbare Defibrillatoren (ICDs) und Medikamente-freisetzende Koronarstents (Drug eluting stents - DES) entwickelt. 


\subsection{Kostensensible Leitlinie zum Einsatz eines implantierbaren Defibrillators (ICDs) - Kurzfassung}

\subsubsection{Krankheitsbild und Behandlungsmöglichkeiten}

Zu den häufigsten Todesursachen im Erwachsenenalter gehört der plötzliche Herztod aufgrund einer koronaren Herzerkrankung (Jung et al. 2006; Priori et al. 2002), bei der die Herzkranzgefäße, die das Herz mit Sauerstoff versorgen, verengt sind. Die Ablagerung von Fetten, Bindegewebe und Kalk in der Gefäßwand führt zu einer Engstelle mit Beeinträchtigung der Blutzufuhr. Klinische Manifestationen der koronaren Herzerkrankung sind u.a. die Angina pectoris, der Herzinfarkt, Herzrhythmusstörungen sowie die Herzinsuffizienz.

Als plötzlichen Herztod definiert man einen unerwarteten natürlichen Tod kardialer Ursache maximal 1 Stunde nach Beginn der Beschwerden. Der plötzliche Herztod tritt bei Erwachsenen mit einer jährlichen Inzidenz von $0,1-0,2 \%$ auf; in Deutschland versterben jährlich ca. 80.000 Menschen am plötzlichen Herztod. Die Hauptursache liegt mit 9o\% in einer tachykarden Herzrhythmusstörung, bei der die Herzfrequenz auf über 100 Herzschläge pro Minute ansteigt. Beim Kammerflimmern zuckt der Herzmuskel unkoordiniert mit einer so hohen Frequenz, dass die Pumpleistung des Herzens nicht mehr ausreicht, um den Körper mit Sauerstoff zu versorgen (Antz u. Kuck 2007). Andere Herzrhythmusstörungen erzeugen eine Bradykardie, d.h. eine Verlangsamung der Herzfrequenz auf weniger als 6o Herzschläge pro Minute.

Neben einer medikamentösen Therapie können Herzrhythmusstörungen mit einem Herzschrittmacher behandelt werden, der die Herzfrequenz steuert, oder mit einem implantablen Kardioverter/Defibrillator (ICD), der neben der Steuerung der Herzfrequenz bei schwerwiegenden Rhythmusstörungen durch die Abgabe eines Stromstoßes versucht, die Herzrhythmusstörung zu durchbrechen und damit den plötzlichen Herztod zu verhindern (Jung et al. 2006; Schwab u. Lüderitz 2007).

\subsubsection{Effektivität eines ICDs}

Das Primärziel einer ICD-Therapie besteht darin, das Leben des Patienten durch die Verhinderung des plötzlichen Herztodes zu verlängern. Die Sekundärziele umfassen die Erhöhung der Lebensqualität und die Senkung des Morbiditätsrisikos (Jung et al. 2006). Nach eigenen Berechnungen auf der Grundlage der SCD-HeFT-Studie von Bardy et al. (2005) verringert sich für Patienten mit einer linksventrikulären Ejektionsfraktion (LVEF) ${ }^{9} \leq 35 \%$ bei der Behandlung mit einem ICD im Vergleich zur medikamentösen Behandlung mit Amiodaron das

9 Bei der linksventrikulären Ejektionsfraktion (LVEF) handelt es sich um ein echokardiographisch ermitteltes Maß für die Pumpfunktion der linken Herzkammer, mit dem man die Fähigkeit des Herzens zur Sauerstoffversorgung des Körpers einschätzen kann. 
6.2 Kostensensible Leitlinie zum Einsatz eines implantierbaren Defibrillators (ICDs) Kurzfassung

Sterblichkeitsrisiko über 5 Jahre um 6,45 Prozentpunkte; dies bedeutet eine Reduktion des Risikos von 28,4\% mit Amiodaron auf 21,95\% mit ICD.

Die medizinische Leitlinie der European Society of Cardiology (ESC) empfiehlt im Rahmen der Primärprävention für herzinsuffiziente (herzmuskelschwache) Patienten mit linksventrikulärer Ejektionsfraktion (LVEF) $\leq 35 \%$ den Einsatz von ICDs unabhängig von der kardialen Grunderkrankung (Dickstein et al. 2008).

\subsubsection{Kosteneffektivität der ICDs ${ }^{10}$}

Der Modellierung von Sanders et al. (2001) zufolge hängt die Kosteneffektivität eines ICDs wesentlich von der Einschränkung der Pumpfunktion des Herzens ab. Bei einer LVEF $\leq 30 \%$ betragen die zusätzlichen Kosten für ein hinzugewonnenes qualitätskorrigiertes Lebensjahr (QALY) ${ }^{11}$ beim Einsatz eines ICDs gegenüber medikamentöser Therapie (Amiodaron) etwa 62.0oo Euro, bei einer LVEF zwischen $31 \%$ und $40 \%$ etwa 168.000 € und bei einer LVEF > 40\% etwa $479.000 €$. Bei einer stärker eingeschränkten Pumpfunktion des Herzens ist der therapeutische Vorteil eines ICDs gegenüber Amiodaron größer und damit das Verhältnis von Kosten zu Effekten günstiger. Tabelle 28 bietet eine Übersicht, wie sich in Abhängigkeit von der Pumpfunktion des Herzens (1) das Sterberisiko der Patienten (als Maß für die Lebensbedrohlichkeit des Krankheitszustands), (2) die Wirksamkeit von ICD und Amiodaron und (3) die Kosteneffektivität eines ICDs im Vergleich zu Amiodaron unterscheiden.

\section{Tab. 28 Sterberisiko der Patienten, Wirksamkeit von ICD und Amiodaron und Kosteneffektivität eines ICDs im Vergleich zu Amiodaron (Quelle: Modellierung von Sanders et al. 2001) (Währungskonversion mit OECD-Kaufkraftparitäten 2007 und auf Tausender gerundet.)}

\begin{tabular}{|c|c|c|c|c|}
\hline & \multicolumn{3}{|c|}{ Linksventrikuläre Ejektionsfraktion (LVEF) } \\
\hline & & $\leq 30 \%$ & $31 \%-40 \%$ & $>40 \%$ \\
\hline \multicolumn{2}{|c|}{ Sterblichkeitsrate nach 3 Jahren } & $34,5 \%$ & $20,6 \%$ & $8,6 \%$ \\
\hline \multirow{3}{*}{$\begin{array}{l}\text { Effektivität in } \\
\text { QALYs }\end{array}$} & ICD & 6,23 & 7,87 & 10,29 \\
\hline & Amiodaron & 5,71 & 7,69 & 10,23 \\
\hline & Differenz & 0,52 & 0,08 & 0,06 \\
\hline \multicolumn{2}{|c|}{$\begin{array}{l}\text { Zusatzkosten in } € \text { pro QALY bei Einsatz } \\
\text { eines ICD statt Amiodaron }\end{array}$} & 62.000 & 168.000 & 479.000 \\
\hline
\end{tabular}

10 Die verfügbare Evidenz zur Kosteneffektivität weist Widersprüche auf, lässt jedoch Tendenzen erkennen, die im Folgenden exemplarisch anhand der Modellierung von Sanders et al. aufgezeigt werden (2001). Berichtet werden hier Ergebnisse für die Annahme einer mittleren Wirksamkeit von ICD und Amiodarone.

11 Die qualitätskorrigierten Lebensjahre (quality adjusted life-years, QALYs) sind ein Maß für die Wirksamkeit medizinischer Verfahren, das Auswirkungen sowohl auf die Restlebenserwartung als auch auf die Lebensqualität erfasst. Bei einer eingeschränkten Lebensqualität ist die Anzahl der hinzugewonnen QALYs geringer als die Anzahl der hinzugewonnenen (unkorrigierten) Lebensjahre. 


\subsubsection{Kostensensible Einschränkung der Indikation}

Auf der Grundlage der Daten zur Kosteneffektivität eines ICDs gegenüber der medikamentösen Behandlung mit Amiodaron kann nun die Indikation wie folgt eingeschränkt werden: Bei einer LVEF von über 30\% liegen Zusatzkosten einer ICD-Implantation bei über $150.000 €$ pro gewonnenem qualitätsadjustierten Lebensjahr (QALY). Die Implantation eines ICD sollte nach der hier vorliegenden Kostensensiblen Leitlinie nur bei einer LVEF $\leq 30 \%$ von der gesetzlichen Krankenkasse übernommen werden, d.h. bei denjenigen Patienten, die eine stärker eingeschränkte Pumpfunktion des Herzen und einen größeren Nutzengewinn ( 0,52 QALYs) von einem ICD haben. Patienten mit einer LVEF $>30 \%$ sind weniger schwer erkrankt, haben ein niedrigeres Sterblichkeitsrisiko und einen deutlich geringeren Nutzengewinn von einem ICD ( $\leq 0,08$ QALYs) und können ggf. medikamentös mit Amiodaron behandelt werden.

Tabelle 29 zeigt die Kostensensible Einschränkung der Indikation im Vergleich zur Empfehlung in der klinischen Leitlinie:

\section{Tab. 29 Kostensensible Einschränkung der Indikation des ICD im Vergleich zur Empfehlung in der klinischen Leitlinie}

\begin{tabular}{|lcccc}
\hline LVEF & $\leq 30 \%$ & $31 \%-35 \%$ & $36 \%-40 \%$ & $>40 \%$ \\
\hline Empfehlung klinische Leitlinie & $\checkmark$ & $\checkmark$ & $\mathbf{x}$ & $\mathbf{x}$ \\
\hline $\begin{array}{l}\text { Empfehlung Kostensensible } \\
\text { Leitlinie (KSLL) }\end{array}$ & $\checkmark$ & $\mathbf{x}$ & $\mathbf{x}$ & $\mathbf{x}$ \\
\hline
\end{tabular}

\section{Anmerkung}

Die hier vorgestellte Kostensensible Leitlinie präsentiert exemplarisch ein mögliches Szenario und basiert nicht auf einer vollständigen Literaturdarstellung.

\section{Literatur}

Antz M, Kuck K-H (2007) Plötzlicher Herztod bei „Herzgesunden“. Herz 32(3), 183

Bardy GH, Lee KL, Mark DB, Poole JE, Packer DL, Boineau R, Domanski M, Troutman C, Anderson J, Johnson G, McNulty SE, Clapp-Channing N, Davidson-R LD, Fraulo ES, Fishbein DP, Luceri RM, Ip JH, for the Sudden Cardiac Death in Heart Failure Trial (SCD-HeFT) Investigators (2005) Amiodaron or an Implantable Cardioverter-Defibrillator for Congestive Heart Failure. N Engl I Med 352, 225-237

Dickstein K, Cohen-Solal A, Filippatos G, McMurray IJV, Ponikowski P, Poole-Wilson PA, Strömberg A, van Veldhuisen DJ, Atar D, Hoes AW, Keren A, Mebazaa A, Nieminen M, Priori SG, Swedberg K (2008) ESC Guidelines for the diagnosis and treatment of acute and chronic heart failure 2008. European Heart Journal 29, 2388-2442

Jung W, Andresen D, Block M, Bocker D, Hohnloser SH, Kuck KH, Sperzel J, Deutsche Gesellschaft für Kardiologie, Herz- und Kreislaufforschung e.V. (2006) Leitlinien zur Implantation von Defibrillatoren. Clin Res Cardiol 95, 696-708 
6.2 Kostensensible Leitlinie zum Einsatz eines implantierbaren Defibrillators (ICDs) Kurzfassung

Priori SG, Aliot E, Blømstrom-Lundqvist C, Bossaert L, Breithardt G, Brugada P, Camm JA, Cappato R, Cobbe SM, Di Mario C, Maron B|, McKenna WI, Pedersen AK, Ravens U, Schwartz PI, Trusz-Gluza M, Vardas P, Wellens HIJ, Zipes DP (2002) Task Force on Sudden Cardiac Death, European Society of Cardiology, Summary of Recommendations. Europace 4, 3-18

Sanders GD, Hlatky MA, Every NR, McDonald KM, Heidenreich PA, Parsons LS, Owens DK (2001) Potential CostEffectiveness of Prophylactic Use of the Implantable Cardioverter Defibrillator or Amiodaron after Myocardial Infarction. Ann Intern Med 135, 870-883

Schwab 10, Lüderitz B (2007) Indikationen für den implantierbaren Cardioverter/Defibrillator (ICD). Internist 48, $715-726$ 


\subsection{Kostensensible Leitlinie zum Einsatz von Medikamente- freisetzenden Koronarstents (DES) - Kurzfassung}

\subsubsection{Krankheitsbild und Behandlungsmöglichkeiten}

Bei der koronaren Herzkrankheit führt die Ablagerung von Fetten, Bindegewebe und Kalk in der Gefäßwand (Arteriosklerose) zu einer Engstelle der Herzkrankgefäße, die die Blutzufuhr und damit die Sauerstoffversorgung des Herzens beeinträchtigt. Klinische Manifestationen der koronaren Herzkrankheit sind u.a. die Angina pectoris, der Herzinfarkt, Herzrhythmusstörungen sowie die Herzinsuffizienz (Classen et al. 2004).

Ein wesentlicher Bestandteil der Therapie bei Angina pectoris und Herzinfarkten ist die Revaskularisierung (Wiedereröffnung) signifikant verengter bzw. verschlossener Herzkranzarterien mittels perkutaner koronarer Intervention (PCI). Bei der PCI wird ein Katheter mit Dilatationsballon meist über eine Arterie des Oberschenkels oder Oberarms bis zu den Koronararterien vorgeschoben. Dies geschieht in der Regel unter Lokalanästhesie in einem stationären Aufenthalt von 1-3 Tagen. Das Risiko, dabei in der Klinik zu versterben, liegt bei Patienten mit stabiler Angina pectoris bei o,4\%, das Auftreten eines schweren kardialen oder zerebrovaskulären Ereignisses (Herzinfarkt, Schlaganfall, transitorische ischämische Attacke, Tod) bei o,7\% (Bundesgeschäftsstelle für Qualitätssicherung 2007). Jährlich werden in Deutschland ca. 270.00o PCI durchgeführt, davon etwa $86 \%$ mit Koronarstents (van Burren u. Horstkotte 2008). Dabei soll ein kleines Metall-Gittergerüst in Röhrchenform den erneuten Gefäßverschluss verhindern.

Nach PCI tritt jedoch in 10-20\% der Fälle und bis zu 50\% bei Patienten mit erhöhtem Risiko eine erneute Verengung des behandelten Gefäßabschnitts durch die Proliferation glatter Muskelzellen auf, die in der Regel eine erneute Revaskularisierung erfordert. Medikamente-freisetzende Koronarstents (drug eluting stents - DES) enthalten antiproliferative Wirkstoffe, die die Wachstumsstimulierung der glatten Muskelzellen unterdrücken. Sie senken dadurch das Risiko für eine erneute Verengung im Vergleich zu konventionellen Metallstents (bare metal stents - BMS) deutlich. Um die erhöhte Gefahr von Stentthrombosen (Blutgerinnsel im Stent) durch den verlangsamten Heilungsprozess bei DES zu reduzieren, ist eine verlängerte Gabe antithrombotischer Medikamente (Acetylsalicylsäure plus Clopidogrel) erforderlich. Im zweiten Jahr nach der Intervention wurden bei DES leicht erhöhte Raten an Stentthrombosen beobachtet, die zu Herzinfarkten und Todesfällen führen können. Insgesamt war das Risiko für Myokardinfarkte und Todesfälle über einen Beobachtungszeitraum von 4 Jahren jedoch nicht statistisch signifikant höher als bei BMS (Stone et al. 2007). DES sind erheblich teurer als BMS. 
6.3 Kostensensible Leitlinie zum Einsatz von Medikamente-freisetzenden Koronarstents (DES) - Kurzfassung

\subsubsection{Effektivität der DES}

Das britische National Institute for Health and Clinical Excellence (NICE) veröffentlichte im Juli 2008 eine Kostensensible Leitlinie zum Einsatz von DES in Großbritannien (NICE 2008). Eine systematische Literaturübersicht mit einer Metaanalyse von 17 randomisierten kontrollierten Studien ergab, dass DES erneute Revaskularisationen um nahezu drei Viertel im Vergleich zu BMS reduzieren konnten. Beispiel: Wenn bei 12 von 100 Patienten mit BMS eine erneute Revaskularisierung durchgeführt werden muss, ist dies nur bei 3 von $100 \mathrm{~Pa}-$ tienten mit DES erforderlich. Dieser zusätzliche Nutzen von DES im Vergleich zu BMS war nach einem Jahr zu sehen und ein signifikanter Unterschied blieb auch nach 3 Jahren erhalten (NICE 2008; Hill et al. 2007). Kein Unterschied fand sich in der Mortalität (Sterblichkeit). Der zusätzliche Nutzen ist für alle Patientengruppen belegt, die in randomisierten klinischen Studien untersucht wurden.

Bei Patienten mit erhöhtem Risiko für eine Restenose aufgrund eines kleinen Gefäßdurchmessers (<3 mm) oder einer längeren Läsion (> $15 \mathrm{~mm})$ ist jedoch die absolute Reduktion des Risikos einer Restenose und damit der zusätzliche Nutzen größer. Beispielsweise benötigten in der TAXUS IV-Studie ca. 8 von 100 Patienten mit langen Läsionen $>20 \mathrm{~mm}$ in der DES-Gruppe eine erneute Revaskularisation, in der BMS-Gruppe hingegen ca. 24 von 100 Patienten, d.h. bei 100 Patienten können 16 Eingriffe durch den Einsatz von DES vermieden werden (Bakhai et al. 2006). Bei Patienten mit Läsionslängen $\leq 20 \mathrm{~mm}$ hatten ca. 6 von 100 Patienten in der DES-Gruppe und ca. 15 von 100 Patienten in der BMS-Gruppe eine erneute Revaskularisation, d.h. hier können bei 100 Patienten durch den Einsatz von DES nur 9 Revaskularisierungen vermieden werden. Für Patienten mit kleinen Gefäßen $(<3,0 \mathrm{~mm})$ betrugen die durch DES vermeidbaren Revaskularisationen 10 pro 100 gegenüber 8 pro 100 bei Patienten mit größeren Gefäßen ( $\geq 3 \mathrm{~mm})$.

\subsubsection{Kosteneffektivität der DES}

Die Kosteneffektivität von DES im Vergleich zu BMS im britischen Gesundheitssystems wurde anhand eines gesundheitsökonomischen Modells ermittelt. Dabei wurden die Kosteneffektivitätsverhältnisse in Abhängigkeit der Risikofaktoren für Restenosen (kleine Gefäße und lange Läsionen) und der Preisdifferenz zwischen DES und BMS berechnet. In Tabelle 30 sind die Kosteneffektivitätsverhältnisse in Abhängigkeit von der Patientengruppe und der Preisdifferenz zwischen DES und BMS angegeben. Die Werte beschreiben die zusätzlichen Kosten, die bei Verwendung von DES im Vergleich zu BMS für ein zusätzlich gewonnenes qualitätsadjustiertes Lebensjahr (QALY) aufgebracht werden müssen. 
Tab. 30 Kosteneffektivitätsverhältnisse in zusätzlichen Euro pro gewonnenem qualitätsadjustiertem Lebensjahr (QALY) (Die Originalwährung britische Pfund wurde anhand von OECD-Kaufkraftparitäten von 2007 in Euro konvertiert und auf Tausender gerundet.)

\begin{tabular}{lcc} 
& \multicolumn{2}{c}{ Preisdifferenz DES - BMS } \\
& $400 €$ & $800 €$ \\
\hline alle Patienten & $98.000 € /$ QALY & $227.000 € /$ QALY \\
\hline Patienten mit langen Läsionen $(>15 \mathrm{~mm})$ & $62.000 € /$ QALY & $167.000 € /$ QALY \\
\hline Patienten mit kleinen Gefäßen $(<3 \mathrm{~mm})$ & $33.000 € /$ QALY & $126.000 € /$ QALY \\
\hline
\end{tabular}

Zentrale Einflussfaktoren in diesem Modell sind

- die Revaskularisationsraten mit BMS in der gesamten Population aller Patienten, die einen Stent erhalten,

- die relative Reduktion des Risikos für eine erneute Revaskularisation mit DES,

- die durchschnittliche Anzahl Stents pro Patient,

- der Nutzwert in QALY für eine vermiedene Revaskularisation,

- die verlängerte zusätzliche Gabe von Clopidogrel bei DES sowie

- die Marktpreise für DES und BMS.

In der NICE-Empfehlung wurde die Evidenz zur relativen Risikoreduktion von Revaskularisationen von DES und zu deren Sicherheit dem systematischen Literaturreview (NICE 2008) entnommen. Die Daten zu den übrigen Parametern beruhen im Wesentlichen auf britischen PCI-Registern sowie Angaben von klinischen Experten.

\subsubsection{Kostensensible Einschränkung der Indikation}

Die Kosteneffektivität des Einsatzes von DES kann verbessert werden, indem man die Indikation auf die Patientengruppen mit größerem Zusatznutzen, d.h. Patienten mit kleinen Gefäßen und langen Läsionen beschränkt. Das resultierende Kosteneffektivitätsverhältnis ist nach Auffassung des NICE aber nur dann akzeptabel, wenn man zusätzlich den Preisunterschied zwischen DES und BMS begrenzt. In Deutschland könnte der Spitzenverband Bund der Krankenkassen nach $\mathbb{3} 3$ Abs. 2a SGB V einen entsprechenden Höchstbetrag für die Vergütung der DES festlegen. Die Kosten für den Einsatz von DES bei der perkutanen Koronarintervention zur Behandlung der koronaren Herzkrankheit würden demzufolge nur dann übernommen, wenn

- das zu behandelnde Zielgefäß einen Durchmesser von weniger als $3 \mathrm{~mm}$ hat oder die Läsion länger als $15 \mathrm{~mm}$ ist und

- der Preisunterschied zwischen DES und BMS nicht mehr als 400 Euro beträgt. 
6.3 Kostensensible Leitlinie zum Einsatz von Medikamente-freisetzenden Koronarstents (DES) - Kurzfassung

\section{Literatur}

Bakhai A, Stone GW, Mahoney E, Lavelle TA, Shi C, Berezin RH, Lahue BJ, Clark MA, Lacey MI, Russell ME, Ellis SG, Hermiller JB, Cox DA, Cohen DI, Investigators T-I (2006) Cost effectiveness of paclitaxel-eluting stents for patients undergoing percutaneous coronary revascularization: results from the TAXUS-IV Trial. I Am Coll Cardiol 48, 253-261

Bundesgeschäftsstelle für Qualitätssicherung (2007) BQS-Bundesauswertung 2007. Qualitätsindikator 6: InHospital-Letalität. URL: http://www.bqs-outcome.de/2007/ergebnisse/leistungsbereiche/PCI/buaw/ index_html/0010_QI_In-Hospital-Letalitaet.html (Zugriff am 21.04.2015)

Classen M, Diehl V, Kochsiek K (2004) Innere Medizin. Urban \& Schwarzenberg München

Hill RA, Boland A, Dickson R, Dundar Y, Haycox A, McLeod C, Mujica Mota R, Walley T, Bagust A (2007) Drug-eluting stents: a systematic review and economic evaluation. Health Technology Assessment 11(46), 1-242

National Institute for Health and Care Excellence (NICE) (2008) NICE technology appraisal guidance 152. Drugeluting stents for the treatment of coronary artery disease (part review of NICE technology appraisal guidance 71). URL: www.nice.org.uk/TA152 (Zugriff am 21.04.2015)

Stone G, Moses I, Ellis S, Schofer I, Dawkins K, Morice M-C, Colombo A, Schampaert E, Grube E, Kirtane A, Cutlip D, Fahy M, Pocock S, Mehran R, Leon M (2007) Safety and Efficacy of Sirolimus- and Paclitaxel-Eluting Coronary Stents. N Engl I Med 356, 998-1008

van Burren F, Horstkotte D (2008) 22. Bericht über die Leistungszahlen der Herzkatheterlabore in der Bundesrepublik Deutschland. Eine Erhebung mit Unterstützung der Kommission für Klinische Kardiologie und der Arbeitsgruppen Interventionelle Kardiologie (für die ESC) und Angiologie der deutschen Gesellschaft für Kardiologie - Herz- und Kreislaufforschung über das Jahr 2005. Kardiologe 2, 320-324 


\subsection{Kostensensible Leitlinien (KSLL): Ein Instrument zur Ausgabensteuerung im Gesundheitswesen - Einführung für medizinische Laien}

\subsubsection{Warum brauchen wir Instrumente zur Steuerung der Gesundheits- ausgaben?}

Die Gesundheitsausgaben steigen trotz aller gesundheitspolitischen Sparbemühungen weiter. Die finanzielle Situation der Gesetzlichen Krankenversicherung (GKV) in Deutschland dürfte sich auch in den kommenden Jahren kaum entschärfen: Neue, häufig sehr teure Diagnose- und Behandlungsverfahren werden die Ausgaben weiter in die Höhe treiben. Gleichzeitig werden die Menschen immer älter und leiden vielfach an mehreren chronischen Erkrankungen, was den Kostendruck im Gesundheitswesen verschärft. Die aktuellen Diskussionen über drohende Defizite bei den Krankenkassen und mögliche Beitragserhöhungen machen es deutlich: Wir brauchen Instrumente, um die Ausgaben der Gesetzlichen Krankenversicherung (GKV) in Deutschland besser kontrollieren zu können. Das Bundesministerium für Bildung und Forschung (BMBF) finanziert seit 2006 ein Forschungsprojekt, den Forschungsverbund „Allokation“, in dem Wissenschaftler aus den Bereichen Ethik, Ökonomie und Recht gemeinsam untersuchen, wie die Ausgaben in der GKV in einer medizinisch vernünftigen, wirtschaftlichen und vor allem ethisch wie rechtlich vertretbaren Art und Weise kontrolliert werden können.

\subsubsection{Was sind „Kostensensible Leitlinien“?}

Wenn im Gesundheitswesen gespart werden muss - so die Überzeugung der beteiligten Wissenschaftler -, sollte es hierfür klare Vorgaben geben, die bestimmen, welcher Patient in welcher Situation welche medizinischen Maßnahmen von der Krankenkasse erstattet erhält. Bislang gibt es wenige Vorschläge, wie solche Vorgaben zur Ausgabensteuerung im Gesundheitswesen aussehen könnten. Der Forschungsverbund „Allokation“ hat in den letzten drei Jahren hierfür ein Modell entwickelt, die Kostensensiblen Leitlinien, abgekürzt mit „KSLL“. Diese Leitlinien geben dem behandelnden Arzt vor, welche Patienten eine teure Maßnahme erhalten sollen und welche Patienten auf eine günstigere ausweichen müssen. Dies hat den Vorteil, dass der Arzt nicht im Einzelfall jedes Mal neu entscheiden muss, welcher Patient welche Behandlung erhält, alle gesetzlich versicherten Patienten werden - entsprechend der Vorgaben - gleich behandelt. 


\subsubsection{Welche Überlegungen liegen den Kostensensiblen Leitlinien zugrunde?}

Wenn im Gesundheitswesen gespart werden muss, sollte man dort ansetzen, wo es den Patienten am wenigsten weh tut: Man sollte auf diejenigen Maßnahmen verzichten, die dem Patienten bei hohen Kosten nur einen geringen Vorteil (Zusatznutzen) gegenüber einer billigeren Alternative bieten. Mit anderen Worten: Der Patient, der auf die günstigere Maßnahme ausweichen muss, hat davon einen vergleichsweise geringen Nachteil. Mit den dadurch freiwerdenden Geldern können dann teure Maßnahmen finanziert werden, die Patienten dringend benötigen und für die es keine Alternativen gibt.

\subsubsection{Wie werden Kostensensible Leitlinien erstellt?}

Es ist sinnvoll, für die Entwicklung einer Kostensensiblen Leitlinie eine Erkrankung auszuwählen, bei der es verschiedene Behandlungsmöglichkeiten gibt, die sich im Preis und in der Wirksamkeit unterscheiden. Um herauszufinden, welche Behandlungsmaßnahmen besser oder schlechter sind, werden zunächst die Ergebnisse wissenschaftlicher Studien zusammengetragen, die die Wirksamkeit und den Nutzen der Behandlungsmaßnahmen untersucht haben. Wenn die Untersuchungsergebnisse zeigen, dass nicht alle Patienten in gleicher Weise von einer teuren Behandlungsmöglichkeit - im Vergleich zur günstigeren Alternative - profitieren, kann eine Kostensensible Leitlinien (KSLL) erstellt werden. Die Leitlinie gibt dem Arzt vor, welche Patienten die teure und welche die günstigere Behandlung erhalten sollen: Diejenigen Patienten, die einen größeren Vorteil von der teureren Behandlung haben, erhalten das teurere Verfahren; diejenigen Patienten, die weniger von ihr profitieren würden, müssen auf die günstigere Behandlungsmöglichkeit ausweichen. Es soll also bei denjenigen Patienten gespart werden, die ein „kleineres Opfer“ erbringen müssen, wenn sie auf die günstigere und etwas weniger wirksame Behandlungsmöglichkeit ausweichen müssen. Im Gegenzug soll auf diese Weise sichergestellt werden, dass Patienten weiterhin eine teure medizinische Maßnahme von der Krankenkasse erstattet bekommen, wenn sie diese dringend benötigen, d.h. wenn sie ihnen einen großen gesundheitlichen Vorteil bietet.

\subsubsection{Wie könnten die Leitlinien im Gesundheitswesen eingesetzt werden?}

In der Gesetzlichen Krankenversicherung (GKV) könnten die Kostensensiblen Leitlinien zum Beispiel vom obersten Gremium der Selbstverwaltung, d.h. vom Gemeinsamen Bundesausschuss (G-BA), als grundsätzlich verbindliche Vorgaben erlassen werden. Sie würden den Ärzten vorgeben, welche diagnostischen und therapeutischen Maßnahmen auf Kosten der Krankenkassen 
durchgeführt werden können. Nur in begründeten Ausnahmefällen kann der Arzt von diesen Vorgaben abweichen.

Um Stärken und Schwächen dieser Instrumente zur Ausgabensteuerung besser einschätzen zu können, wurden im Forschungsverbund zwei Kostensensible Leitlinien als Modelle erarbeitet: Zum einen für den Einsatz von implantierbaren Defibrillatoren (ICDs) bei schweren Herzrhythmusstörungen und zum anderen für den Einsatz von Medikamente-freisetzenden Koronarstents (Drug eluting stents - DES). 
6.5 Kostensensible Leitlinie zum Einsatz von Medikamente-freisetzenden Koronarstents:

\subsection{Kostensensible Leitlinie zum Einsatz von Medikamente- freisetzenden Koronarstents: Kurzfassung für medizinische Laien}

\subsubsection{Was ist eine koronare Herzkrankheit?}

Bei der koronaren Herzkrankheit führt die Ablagerung von Fetten, Bindegewebe und Kalk in der Gefäßwand zu einer Verengung der Blutgefäße des Herzens. Dies beeinträchtigt die Blutzufuhr und damit die Sauerstoffversorgung des Herzens. Die Patienten leiden typischerweise unter einem Engegefühl und Schmerzen über der Brust (sog. Angina pectoris). Wenn die Erkrankung fortschreitet, können sich ein Herzinfarkt (Verschluss eines Blutgefäßes des Herzens), Herzrhythmusstörungen sowie eine verschlechterte Pumpfunktion des Herzens entwickeln.

\subsubsection{Wie kann man die koronare Herzkrankheit behandeln?}

Eine wichtige Behandlungsmöglichkeit der koronaren Herzerkrankung ist die Wiedereröffnung von verengten oder verschlossenen Blutgefäßen des Herzens. Dabei wird ein Katheter - unter örtlicher Betäubung - mit einem Ballon über eine Arterie des Oberschenkels oder Oberarms bis zu den verengten oder verschlossenen Blutgefäßen des Herzens vorgeschoben. Durch Aufblasen des Ballons wird das verengte Blutgefäß erweitert und die Blutversorgung des Herzens wieder verbessert. Die Patienten bleiben für den Eingriff in der Regel 1-3 Tage im Krankhaus. Das Risiko dabei im Krankhaus zu versterben, liegt bei Patienten in einem stabilen Zustand bei o,4\%; bei o,7\% der Patienten tritt eine schwerwiegende Komplikation am Herzen oder den Blutgefäßen des Gehirns auf (Herzinfarkt, Schlaganfall, vorübergehender Ausfall von Gehirnfunktionen, Tod). Pro Jahr werden in Deutschland etwa 270.0oo Herzkatheter-Eingriffe durchgeführt. Bei etwa 86\% der Patienten wird ein sog. Koronarstent eingesetzt: Ein kleines Metallgitter-Röhrchen - vergleichbar mit einem rund gewickelten Maschendrahtzaun - wird in das wieder eröffnete Gefäß eingelegt und soll einen erneuten Gefäßverschluss verhindern.

Nach einem Herzkatheter-Eingriff tritt jedoch in 10-20\% der Fälle eine erneute Verengung des behandelten Gefäßabschnitts auf, bei Patienten mit einem erhöhten Risiko in bis zu 50\% der Fälle. In der Regel ist dann eine erneute Herzkatheter-Untersuchung mit einer Wiedereröffnung des Gefäßes erforderlich. Um die erneute Verengung der Gefäße zu verhindern wurden Koronarstents sog. drug eluting stents (DES) - entwickelt, die bestimmte Medikamente freisetzen. Diese Wirkstoffe sollen verhindern, dass Muskelzellen in das Gefäß einwachsen und es erneut verschließen. Sie senken dadurch das Risiko für eine erneute Verengung deutlich im Vergleich zu den reinen Metallstents - sog. bare metal stents (BMS). Da sich bei Medikamente-freisetzenden Stents eher Blutgerinnsel im Röhrchen bilden, ist eine verlängerte Gabe von Medikamenten erforderlich, die die Blutgerinnung hemmen (Acetylsalicylsäure plus Clo- 
pidogrel). Medikamente-freisetzende Stents sind erheblich teurer als die reinen Metallgitter-Röhrchen.

\subsubsection{Welchen Vorteil bieten Medikamente-freisetzende Stents?}

In Großbritannien wurden die Erkenntnisse aus 17 wissenschaftlichen Untersuchungen analysiert und zusammengefasst. Demnach können Medikamente-freisetzende Stents (DES) die Anzahl erneuter Herzkathetereingriffe zur Wiedereröffnung des Gefäßes um nahezu drei Viertel im Vergleich zu reinen Metallstents (BMS) verringern. Dies sei an einem Beispiel erläutert: Wenn bei 12 von 100 Patienten mit einem Metallstent (BMS) eine Wiedereröffnung durchgeführt werden muss, ist dies nur bei 3 von 100 Patienten mit einem Medikamenten-Stent (DES) erforderlich. Dieser zusätzliche Nutzen der Medikamenten-Stents war nach einem Jahr zu sehen und ein deutlicher (signifikanter) Unterschied blieb auch nach 3 Jahren erhalten. Kein Unterschied fand sich hingegen bei der Sterblichkeit. Der zusätzliche Nutzen ist für alle Patientengruppen belegt, die in klinischen Studien untersucht wurden.

\subsubsection{Welche Patienten haben einen größeren, welche einen kleineren Vorteil von den Medikamente-freisetzenden Stents?}

Patienten mit einem kleinen Gefäßdurchmesser $(<3 \mathrm{~mm})$ oder einer längeren Engstelle (>15 mm) haben ein erhöhtes Risiko einer erneuten Verengung des Gefäßes. Bei diesen Patienten kann das Risiko einer erneuten Gefäßverengung durch den Einsatz eines Medikamenten-Stents (DES) in einem größeren Ausmaß verringert werden, bei ihnen ist der zusätzliche Nutzen damit größer. Dies sei wiederum an einem Beispiel verdeutlicht: In einer Studie benötigten etwa 8 von 100 Patienten mit langen Engstellen (> $20 \mathrm{~mm}$ ) eine erneute Gefäßeröffnung, wenn sie einen Medikamenten-Stent (DES) bekommen hatten; in der Gruppe mit einem reinen Metallstent (BMS) waren es hingegen etwa 24 von 100 Patienten. In diesem Fall konnten bei 100 Patienten 16 HerzkatheterEingriffe durch den Einsatz von Medikamenten-Stents (DES) vermieden werden. Bei Patienten mit kürzeren Engstellen $(\leq 20 \mathrm{~mm})$ hatten etwa 6 von 100 Patienten mit Medikamenten-Stent und etwa 15 von 100 Patienten mit Metall-Stent einen erneuten Eingriff zur Wiedereröffnung des Gefäßes. Bei dieser Cruppe können bei 100 Patienten durch den Einsatz von Medikamenten-Stents nur 9 Herzkatheter-Eingriffe vermieden werden. Bei Patienten mit kleinen Gefäßen (<3,o mm) konnten durch die Medikamenten-Stents 10 Herzkatheter-Eingriffe pro 100 Patienten eingespart werden, gegenüber 8 Eingriffen pro 100 bei Patienten mit größeren Gefäßen ( $\geq 3 \mathrm{~mm}$ ). 
6.5 Kostensensible Leitlinie zum Einsatz von Medikamente-freisetzenden Koronarstents: Kurzfassung für medizinische Laien

\subsubsection{Was kostet der Nutzengewinn durch die Medikamenten-Stents (DES)?}

Daten zum Verhältnis von Nutzen (= Vorteil) und Kosten liegen aus dem britischen Gesundheitssystem vor. Dabei wurde das Kosten-Nutzen-Verhältnis in Abhängigkeit vom Risiko eines erneuten Gefäßverschlusses (kleine Gefäße und lange Engstellen) und vom Preisunterschied zwischen dem Medikamenten-Stent (DES) und dem reinen Metallstent (BMS) berechnet. Tabelle 31 zeigt das Kosten-Nutzen-Verhältnis in Abhängigkeit von den Risikofaktoren und dem Preisunterschied. Die Werte beschreiben die zusätzlichen Kosten in Euro, die bei Verwendung eines Medikamenten-Stents im Vergleich zu einem reinen Metallstent für ein hinzu gewonnenes Lebensjahr aufgebracht werden müssen. Dabei wird auch die Lebensqualität berücksichtigt: Ein QALY (englisch: quality adjusted life year) entspricht einem Lebensjahr in perfekter Lebensqualität. Dies sei erneut an einem Beispiel verdeutlicht: Gewinnt ein Patient durch ein Medikament zwei Lebensjahre mit einer um die Hälfte reduzierten Lebensqualität $(=0,5)$ hinzu, entspricht der Nutzengewinn insgesamt o,5 $\mathrm{x}$ 2 Jahre = 1 qualitätsbereinigtes Lebensjahr (QALY).

\section{Tab. 31 Kosten-Nutzen-Verhältnis in zusätzlichen Euro pro gewonnenem qualitätsbereinigtem Lebensjahr (QALY)}

\begin{tabular}{lcc} 
& \multicolumn{2}{c}{ Preisunterschied DES - BMS } \\
& $400 €$ & $800 €$ \\
\hline alle Patienten & $98.000 € /$ QALY & $227.000 € /$ QALY \\
\hline Patienten mit langen Engstellen $(>15 \mathrm{~mm})$ & $62.000 € /$ QALY & $167.000 € /$ QALY \\
\hline Patienten mit kleinen Gefäßen $(<3 \mathrm{~mm})$ & $33.000 € /$ QALY & $126.000 € /$ QALY \\
\hline
\end{tabular}

Aus Tabelle 4 wird deutlich, dass das Verhältnis von Kosten zu Nutzen bei Patienten mit einer langen Engstelle und einem kleinen Gefäßdurchmesser günstiger ist, da die Wahrscheinlichkeit eines erneuten Gefäßverschlusses höher und damit der Vorteil (= Nutzengewinn) eines Medikamenten-Stents größer ist. Natürlich hängt das Kosten-Nutzen-Verhältnis auch von den Preisen der Stents ab: Je größer der Preisunterschied ist, desto mehr muss man für den Nutzengewinn (= die verringerte Anzahl von Herzkatheter-Eingriffen) bezahlen, wenn man von einem Metall-Stent auf einen Medikamenten-Stent umsteigt.

\subsubsection{Wie könnte eine Kostensensible Leitlinie bei der koronaren Herzkrank- heit aussehen?}

Die wissenschaftlichen Studien haben gezeigt, dass Patienten mit kleinen Gefäßen und langen Engstellen mehr von den teuren Medikamenten-Stents (DES) profitieren, weil sie ein höheres Risiko für einen erneuten Gefäßver- 
schluss haben. Wenn man im Bereich der koronaren Herzkrankheit gezielt sparen möchte, könnte man mit einer Kostensensiblen Leitlinie (KSLL) die Anwendung der teuren Medikamente-freisetzenden Stents (DES) auf diejenigen Patienten mit einer koronaren Herzkrankheit beschränken, die einen größeren Vorteil von ihnen haben: Patienten mit kleinen Gefäßen und einer langen Engstelle. Patienten mit größeren Gefäßen und kürzeren Engstellen, die eine niedrigere Wahrscheinlichkeit für einen erneuten Gefäßverschluss haben, würden von ihrer Krankenkasse nur den reinen Metall-Stent bezahlt bekommen. Sie würden zwar auch von den Medikamenten-Stents profitieren, aber in einem geringeren Ausmaß als die Patienten mit einem kleinen Gefäß und einer langen Engstelle.

Zusätzlich könnte der Spitzenverband der Krankenkassen einen Höchstbetrag für die Vergütung der Medikamenten-Stents (DES) festlegen, was zu weiteren Einsparungen führen würde. 